\title{
ON THE NONLINEAR THEORY OF ELASTIC SHELLS UNDER THE KIRCHHOFF HYPOTHESIS*
}

\author{
BY \\ P. M. NAGHDI AND R. P. NORDGREN \\ University of California, Berkeley
}

1. Introduction. Despite several significant contributions, the complete nonlinear bending theory of elastic shells which would include fully general and nonlinear constitutive equations and strain measures is not as yet available. The earliest general investigations are by Synge and Chien [1] and Chien [2] who evidently were the first to adopt the intrinsic approach, thus avoiding direct reference to displacements. These papers of Synge and Chien, which are remarkable for their generality, utilize linear constitutive relations. ${ }^{1}$ More recently, an exact theory of strain for shells and rods, based on the concept of oriented bodies of E. and F. Cosserat, was formulated by Ericksen and Truesdell [4] who, however, did not consider the problem of constitutive equations. ${ }^{2}$ Other developments which also employ linear constitutive relations are founded under the Kirchhoff hypothesis and often contain other approximations. Among these we mention the incomplete treatment of Novozhilov [5; pp. 186-198], E. Reissner's [6, 7] formulation of axisymmetric deformation of shells of revolution, and the more general works of Sanders [8] and Leonard [9]. Both Sanders [8] and.Leonard [9] independently have, through an heuristic argument, proposed the differences of the first fundamental forms and of the second fundamental forms between the deformed and the undeformed middle surface of the shell as measures of deformation in "extension" and "bending," respectively; also, after postulating the existence of a two-dimensional strain energy function for shells dependent only on these two measures of deformation, ${ }^{3}$ they have derived constitutive equations by means of the generalized Hooke's law for the case of finite displacement and infinitesimal strains.

Beginning with the three-dimensional field equations, the present paper is concerned with an exact, complete, and fully general nonlinear theory of elastic shells founded under the Kirchhoff hypothesis. ${ }^{4}$ Since the fully general equations of equilibrium in terms of stress and couple resultants and the appropriate boundary conditions are well known, ${ }^{5}$ the main task confronting us is the determination of suitable strain measures and the derivation of constitutive equations.

${ }^{*}$ Received August 16, 1962. The results presented here were obtained in the course of research sponsored by the Office of Naval Research under Contract Nonr 222(69), Project NR-064-436, with the University of California, Berkeley.

1The work of Chien has been criticized by a number of investigators and has been re-examined by Reiss who discovered several errors in Chien's work. For a brief account of these and related references, see [3].

2The paper of Ericksen and Truesdell [4] also contains an elegant derivation of the general equations of equilibrium for shells via the direct method which is shorter than that given earlier by Synge and Chien [1].

${ }^{3}$ The connection between the strain energy for shells (per unit area of the middle surface) and the corresponding strain energy function in 3-space is not considered in [8] and [9].

4This hypothesis entails the vanishing of transverse shear strains and the transverse normal strain.

${ }^{5} \mathrm{~A}$ satisfactory derivation of the general equations of equilibrium for shells from the stress differential equations of equilibrium in 3 -space is given in [10; Sec. 5]. 
First, with the aid of certain results established recently in [10], a representation of finite strain measures for shells (and plates) is obtained in Sec. 2. This representation of the strain measures, which is exact under the Kirchhoff hypothesis, has a remarkably simple structure and is derived with the aid of Cauchy-Green measures of deformation in 3 -space. Next, with the use of the principle of virtual work and in light of the representation of the strain measures established in Sec. 2, the structure of the fully nonlinear constitutive equations (in the sense of Green) is derived in Sec. 3. These constitutive equations may also be derived by direct integration (with respect to the coordinate along the normal to the deformed middle surface of the shell) of the constitutive relations of the nonlinear theory of hyperelastic materials in 3-space. As this alternate method of derivation is also instructive, it is briefly carried out in Sec. 4, where the reduction to the corresponding results of the linear theory is included. Finally, the nature of kinematic approximations, often adopted in contemporary literature, is discussed in Sec. 5 with special reference to axisymmetric deformation of shells of revolution. The conclusions arrived at are in accord with intuitive expectations and give further support to such approximate theories as that in $[6,7]$, where the displacements are finite but the strains are assumed to be infinitesimal.

The notation employed throughout the paper is essentially that of Ref. [10]. In particular, in order to distinguish between material and spatial descriptions, capital letters and indices (Latin or Greek) are used for the former and lower case letters and indices for the latter. Latin indices will have the range 1, 2, 3, whereas Greek indices with the range 1, 2 will be reserved for subtensors and surface tensors. Also, all letters with a superposed asterisk refer to 3-space.

2. Preliminaries. A representation of finite strain measures for shells. Let $X^{A}$ and $\dot{x}^{i}$ denote the material and spatial curvilinear coordinates referred to a fixed righthanded orthogonal Cartesian system in a Euclidean 3-space and let the motion of the shell be represented by transformation relations of the form

$$
x^{i}=x^{i}\left(X^{A}, t\right) ; \quad\left|x_{, A}^{i}\right|>0,
$$

where comma denotes partial differentiation, $X^{A}$ are the initial coordinates of a generic point of the shell space ${ }^{6}$ and $x^{i}$ the coordinates of the same point at time $t$. Further, let $X^{\boldsymbol{A}}$ and $x^{i}$ be identified with a set of normal coordinates so that the position vectors of a generic point of the shell space in material and spatial frames may be represented as

$$
\begin{aligned}
\mathbf{R}^{*} & =\mathbf{R}\left(X^{\Delta}\right)+X^{3} \mathbf{A}_{3}\left(X^{\Delta}\right) ; \quad \mathbf{R}_{\cdot \Delta}^{*} \cdot \mathbf{A}_{3}=0, \mathbf{A}_{3} \cdot \mathbf{A}_{3}=1, \\
\mathbf{r}^{*} & =\mathbf{r}\left(x^{\alpha}\right)+x^{3} \mathbf{a}_{3}\left(x^{\alpha}\right) ; \quad \mathbf{r}_{, \alpha}^{*} \cdot \mathbf{a}_{3}=0, \mathbf{a}_{3} \cdot \mathbf{a}_{3}=1,
\end{aligned}
$$

where $R$ and $r$ are the position vectors of a generic point of the material and spatial middle surfaces having $\mathbf{A}_{3}$ and $\mathbf{a}_{3}$ as unit normal vectors, respectively.

Now, let us admit the restricted transformation relations

$$
X^{\Delta}=X^{\Delta}\left(x^{\alpha}\right), \quad X^{3}=x^{3} ; \quad\left|X_{, \alpha}^{\Delta}\right|>0
$$

and its inverse. The mapping (2.3) characterizing the deformation of the shell space is equivalent to the Kirchhoff hypothesis according to which normal lines $X^{\Delta}=$ const.

${ }^{6} \mathrm{~A}$ precise definition of a shell is given in [10; Sec. 4]. The shell space refers to the region occupied by the shell which, e.g., with reference to spatial frame, is defined by its middle surface $s$ with boundary $c$ and $\left|x^{3}\right| \leq h_{/ 2}<r_{\mathrm{m} \text { in }} \neq 0$, where $h$ is the thickness of the shell and $r_{\mathrm{m} \text { in }}$ stands for the minimum (local) radius of curvature. 
remain normal to the deformed middle surface and surfaces originally parallel to the undeformed middle surface remain parallel to the deformed middle surface and at the same distance from it. As brought out in [10; Sec. 4], under the Kirchhoff hypothesis, the representation of the displacement vector in the form

$$
\mathbf{u}=\mathbf{r}^{*}-\mathbf{R}^{*}=\mathbf{v}\left(x^{\alpha}\right)+x^{3} \boldsymbol{\beta}\left(x^{\alpha}\right)
$$

is exact and furthermore leads to the following strain measures

$$
\begin{gathered}
2 e_{\alpha \beta}=\mu_{\alpha}^{\lambda}\left(\phi_{\lambda \beta}+x^{3} \kappa_{\lambda \beta}\right)+\mu_{\beta}^{\lambda}\left(\phi_{\lambda \alpha}+x^{3} \kappa_{\lambda \alpha}\right)-a^{\lambda \eta}\left(\phi_{\lambda \alpha}+x^{3} \kappa_{\lambda \alpha}\right)\left(\phi_{\eta \beta}+x^{3} \kappa_{\eta \beta}\right) \\
-\left(\phi_{\alpha}^{3}+x^{3} \kappa_{\alpha}^{3}\right)\left(\phi_{\beta}^{3}+x^{3} \kappa_{\beta}^{3}\right), \quad e_{\alpha 3}=e_{33}=0,
\end{gathered}
$$

where

$$
\begin{gathered}
\boldsymbol{\nabla}=v^{\alpha}\left(x^{\gamma}\right) \mathbf{a}_{\alpha}+w\left(x^{\gamma}\right) \mathbf{a}_{3} \equiv \mathbf{r}\left(x^{\alpha}\right)-\mathbf{R}\left[X^{\Delta}\left(x^{\alpha}\right)\right], \\
\beta=\beta^{\alpha}\left(x^{\gamma}\right) \mathbf{a}_{\alpha}+\beta^{3}\left(x^{\gamma}\right) \mathbf{a}_{3} \equiv \mathbf{a}_{3}\left(x^{\alpha}\right)-\mathbf{A}_{3}\left[X^{\Delta}\left(x^{\alpha}\right)\right], \\
\boldsymbol{\phi}_{. \alpha}^{\beta}=v_{11 \alpha}^{\beta}-b_{\alpha}^{\beta} w, \quad \boldsymbol{\phi}_{\alpha}^{3}=w_{, \alpha}+b_{\alpha \gamma} v^{\gamma}, \\
\boldsymbol{\kappa}_{. \alpha}^{\beta}=\beta_{11 \alpha}^{\beta}-b_{\alpha}^{\beta} \beta^{3}, \quad \kappa_{\alpha}^{3}=\beta_{, \alpha}^{3}+b_{\alpha \gamma} \beta^{\gamma},
\end{gathered}
$$

$\mathbf{a}_{\alpha}=\mathbf{r}_{, \alpha}$ is the surface base vector, $a_{\alpha \beta}$ and $b_{\alpha \beta}$ denote, respectively, the first and the second fundamental forms of the surface, ()$_{1 / \alpha}$ denotes covariant differentiation with respect to $a_{\alpha \beta}$ and

$$
\mu_{\beta}^{\alpha}=\left(\delta_{\beta}^{\alpha}-x^{3} b_{\beta}^{\alpha}\right)
$$

$\delta_{\beta}^{\alpha}$ being the Kronecker symbol.

We now proceed to obtain an alternate representation of strain measures for shells. Denoting the metric tensors in the material and spatial normal coordinate systems by $G_{A B}$ and $g_{i i}$, respectively, and recalling that under the Kirchhoff hypothesis and in normal coordinates, the Cauchy-Green measures of deformation yield [10; Sec. 4]

$$
2 e_{\alpha \beta}=g_{\alpha \beta}-X_{, \alpha}^{\Delta} X_{, \beta}^{\Gamma} G_{\Delta \Gamma}, \quad e_{\alpha 3}=e_{33}=0,
$$

then with the aid of (2.3) and the fact that

$$
g_{\alpha \beta}=\mu_{\alpha}^{\nu} \mu_{\beta}^{\lambda} a_{p \lambda},
$$

the first of (2.9) may be put in the form

$$
2 e_{\alpha \beta}=2{ }_{0} e_{\alpha \beta}+2 x^{3}{ }_{1} e_{\alpha \beta}+2\left(x^{3}\right)^{2}{ }_{2} e_{\alpha \beta},
$$

where

$$
\begin{gathered}
2{ }_{0} e_{\alpha \beta}=a_{\alpha \beta}-X_{, \alpha}^{\Delta} X_{, \beta}^{\Gamma} A_{\Delta \mathrm{\Gamma}}, \\
{ }_{1} e_{\alpha \beta}=-\left[b_{\alpha \beta}-X_{, \alpha}^{\Delta} X_{, \beta}^{\mathrm{\Gamma}} B_{\Delta \mathrm{\Gamma}}\right], \\
2{ }_{2} e_{\alpha \beta}=b_{\lambda \alpha} b_{\beta}^{\lambda}-X_{, \alpha}^{\Delta} X_{, \beta}^{\mathrm{\Gamma}} B_{\Theta_{\Delta}} B_{\Gamma}^{\Theta}
\end{gathered}
$$

and $A_{\Delta \Gamma}$ and $B_{\Delta \Gamma}$ are the dual of $a_{\alpha \beta}$ and $b_{\alpha \beta}$ in the material frame. In addition, comparison of (2.5) and (2.11) furnishes the relations

$$
\begin{aligned}
& 2{ }_{0} e_{\alpha \beta}=\left(\phi_{\alpha \beta}+\phi_{\beta \alpha}\right)-\phi_{\alpha}^{\lambda} \phi_{\lambda \beta}-\phi_{\alpha}^{3} \phi_{\beta}^{3}, \\
& 2{ }_{1} e_{\alpha \beta}=\left(\kappa_{\alpha \beta}+\kappa_{\beta \alpha}\right)-b_{\alpha}^{\lambda} \phi_{\lambda \beta}-b_{\beta}^{\lambda} \phi_{\lambda \alpha}-\phi_{\alpha}^{\lambda} \phi_{\lambda \beta}-\kappa_{\beta}^{\lambda} \phi_{\lambda \alpha}-\kappa_{\alpha}^{3} \phi_{\beta}^{3}-\kappa_{\beta}^{3} \phi_{\alpha}^{3}, \\
& 2{ }_{2} e_{\alpha \beta}=-b_{\alpha}^{\lambda} \kappa_{\lambda \beta}-b_{\beta}^{\lambda} \kappa_{\lambda \alpha}-\kappa_{\alpha}^{\lambda} \kappa_{\lambda \beta}-\kappa_{\alpha}^{3} \kappa_{\beta}^{3} .
\end{aligned}
$$


For future reference, we also recall the material description of strain in the form

$$
2 E_{\Delta \Gamma}=2 x_{, \Delta}^{\alpha} x_{, \Gamma}^{\beta} e_{\alpha \beta}=x_{, \Delta}^{\alpha} x_{, \Gamma}^{\beta} g_{\alpha \beta}-G_{\Delta \Gamma}, \quad E_{\Gamma 3}=E_{33}=0
$$

from which, together with (2.3), follows the dual of (2.11) and (2.12), namely

$$
\begin{aligned}
& 2 E_{\Delta \mathrm{\Gamma}}=2{ }_{0} E_{\Delta \mathrm{\Gamma}}+2 x^{3}{ }_{1} E_{\Delta \mathrm{\Gamma}}+2\left(x^{3}\right)^{2}{ }_{2} E_{\Delta \mathrm{\Gamma}},
\end{aligned}
$$

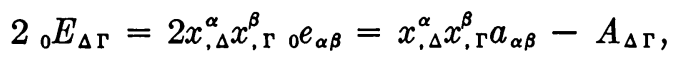

$$
\begin{aligned}
& { }_{1} E_{\Delta \mathrm{r}}=x_{, \Delta}^{\alpha} x_{, \mathrm{\Gamma}{ }_{1} e_{\alpha \beta}}=-x_{, \Delta}^{\alpha} x_{, \mathrm{r}}^{\beta} b_{\alpha \beta}+B_{\Delta \mathrm{r}}, \\
& 2{ }_{2} E_{\Delta \mathrm{\Gamma}}=2 x_{, \Delta}^{\alpha} x_{, \Gamma 2}^{\beta} e_{\alpha \beta}=x_{, \Delta}^{\alpha} x_{, \mathrm{r}}^{\beta} b_{\alpha \lambda} b_{\beta}^{\lambda}-B_{\Delta \Theta} B_{\mathrm{r}}^{\Theta} \text {. }
\end{aligned}
$$

As the structure of the right-hand sides of (2.12) and (2.15) are reminiscent of the first, second, and third fundamental forms of a surface, it is desirable to obtain a further connection between ${ }_{2} e_{\alpha \beta},{ }_{1} e_{\alpha \beta}$, and ${ }_{0} e_{\alpha \beta}$. To this end, we designate the mean and the Gaussian curvatures of the material and spatial middle surface by

$$
\Omega=\frac{1}{2} B_{\Gamma}^{\Gamma}, \quad K=\left|B_{\Delta}^{\Gamma}\right|,
$$

and

$$
\omega=\frac{1}{2} b_{\gamma}^{\gamma}, \quad k=\left|b_{\gamma}^{\gamma}\right|,
$$

respectively, and recall the Hamilton-Cayley theorem for a $2 \times 2$ matrix which, when applied to $b_{\alpha \beta}$, reads

$$
k a_{\alpha \beta}=2 \omega b_{\alpha \beta}-b_{\alpha \lambda} b_{\beta}^{\lambda} .
$$

Substituting (2.12b) into (2.12c), making use of (2.12a), (2.17), and its dual, then after some rearrangement we deduce

$$
{ }_{2} e_{\alpha \beta}=-\left\{\Omega_{1} e_{\alpha \beta}+K_{0} e_{\alpha \beta}-(\omega-\Omega) b_{\alpha \beta}+\frac{1}{2}(k-K) a_{\alpha \beta}\right\}
$$

with the aid of which (2.11) may be written as

$$
e_{\alpha \beta}=\left[1-\left(x^{3}\right)^{2} K\right]_{0} e_{\alpha \beta}+x^{3}\left[1-x^{3} \Omega\right]_{1} e_{\alpha \beta}+\left(x^{3}\right)^{2} r_{\alpha \beta},
$$

where

$$
r_{\alpha \beta}=\left[(\omega-\Omega) b_{\alpha \beta}-\frac{1}{2}(k-K) a_{\alpha \beta}\right] .
$$

It is clear from (2.18) that ${ }_{2} e_{\alpha \beta}$ cannot be expressed entirely in terms of ${ }_{0} e_{\alpha \beta}$ and ${ }_{1} e_{\alpha \beta}$ alone. Hence, according to (2.11), [or equivalently (2.19)], which is exact under the Kirchhoff hypothesis, the strain measures for the shell space, to within rigid motion, are uniquely determined by ${ }_{n} e_{\alpha \beta},(n=0,1,2)$. Although the exact representation (2.11), which includes ${ }_{2} e_{\alpha \beta}$ does not appear to have been noted previously, it may be mentioned that the selection of ${ }_{0} e_{\alpha \beta}$ and ${ }_{1} e_{\alpha \beta}$, as two independent submeasures of deformation, argued on an intuitive basis, has been proposed independently by both Sanders [8] and Leonard [9]. In this connection and with reference to (2.19), it is interesting to ask under what circumstances the strain measures are determined by ${ }_{0} e_{\alpha \beta}$ and ${ }_{1} e_{\alpha \beta}$ alone.

It follows from (2.19b) that $r_{\alpha \beta}$ vanishes if and only if

$$
b_{\alpha \beta}=\frac{1}{2}\left[\frac{k-K}{\omega-\Omega}\right] a_{\alpha \beta} .
$$

${ }^{7}$ Other representations of ${ }_{2} e_{\alpha \beta}$ which, e.g., in addition to ${ }_{0} e_{\alpha \beta}$ and ${ }_{1} e_{\alpha \beta}$, will contain $X_{, \alpha}^{\Delta}$ as well as $A_{\Delta \mathrm{r}}$ and $B_{\Delta \mathrm{r}}$ are possible, but they are not as revealing as that given by (2.18). 
Setting $\frac{1}{2}[k-K / \omega-\Omega]=r^{-1}$, then it may be easily shown by Mainardi-Codazzi relations, namely $b_{\alpha \beta \| \gamma}=b_{\alpha \gamma \| \beta}$, that $r$ is constant and hence $(2.20)$ has the form $b_{\alpha \beta}=r^{-1} a_{\alpha \beta}$. But this in turn demands that the spatial middle surface be spherical with $r$ as the radius of curvature, i.e., $\omega=r^{-1}, k=r^{-2}$, with the aid of which (2.20) also provides the additional restriction

$$
r^{-1}=\Omega \pm\left[\Omega^{2}-K\right]^{1 / 2} .
$$

Clearly, (2.21) is fulfilled if the undeformed middle surface is spherical, i.e., $B_{\Delta \mathrm{r}}=R^{-1} A_{\Delta \mathrm{\Gamma}}, \Omega^{2}=K=R^{-2}$. However, the initial configuration need not be restricted only to a spherical shell and for $\Omega^{2} \neq K$, it follows from $\left(r^{-1}\right)_{, \alpha}=0$ that

$$
\frac{1}{2} K \pm \frac{1}{r} \Omega=\text { const., (say C). }
$$

To obtain more specific information, let (2.22) be referred to principal directions and let $B_{1}^{1}=\alpha B_{2}^{2}$ so that

$$
\left(B_{2}^{2}\right)^{2} \pm \frac{1}{\alpha r}(1+\alpha) B_{2}^{2}-\frac{\mathrm{C}}{\alpha}=0,
$$

the solution of which represents a family of surfaces including the hyperbolic paraboloid.

Thus, under the Kirchhoff hypothesis, the strain measures are given by (2.19a) with $r_{\alpha \beta}=0$ provided the deformed middle surface is spherical and its radus of curvature meets (2.21) for all admissible $\Omega$ and $K$. In particular, with $r_{\alpha \beta}=0$, for an initially flat plate $(\Omega=K=0)$, the deformation is simply plane and, if the undeformed middle surface is spherical, by (2.21) $r=R$, and no deformation is possible.

3. Derivation of the constitutive equations. Utilizing the exact strain measures (2.11), we now proceed to derive general constitutive equations for elastic shells by means of a virtual work principle in 3-space. To avoid unnecessary duplications in the derivation that follows, the boundary conditions are not included.

We recall (see, e.g., Green and Zerna [11]) that during a virtual displacement, the expression for virtual work of the body and surface forces acting on the shell space in equilibrium at time $t$ may be written as

$$
\delta U=\int_{0} \sigma^{i j} \delta e_{i j} d v=\int_{0} \frac{\rho^{*}}{\rho_{0}^{*}} \delta \Sigma^{*} d v,
$$

where $\rho^{*}$ is the mass density at time $t, \rho_{0}^{*}$ is the initial mass density, $\sum^{*}\left(e_{i j}\right)$ is the elastic potential (or the strain energy density) of the shell space, $\sigma^{i j}$ are the spatial components of the stress tensor which satisfies the symmetry condition $\epsilon_{i j k} \sigma^{i k}=0$, $\epsilon_{i, k}$ is the $\epsilon$-system in 3-space, and the element of volume $d v$ at time $t$ is given by

$$
d v=(g / a)^{1 / 2} d s d x^{3},
$$

$d s$ being an element of area of the spatial middle surface.

Now by (2.9), (2.10), and the use of (2.8), we have

$$
2 \delta e_{\alpha \beta}=\delta g_{\alpha \beta}=\delta\left[\mu_{\alpha}^{\lambda} \mu_{\beta}^{\eta} a_{\lambda_{\eta}}\right]=\mu_{\alpha}^{\lambda} \mu_{\beta}^{\eta} \delta a_{\lambda \eta}-x^{3}\left[\mu_{\alpha \lambda} \delta b_{\beta}^{\lambda}+\mu_{\beta \lambda} \delta b_{\alpha}^{\lambda}\right]
$$

${ }^{8}$ Although the virtual work expression in [11] is derived in a convected coordinate system, it is equally valid in an arbitrary spatial frame of reference which is regarded as convected only during a virtual displacement, so that when $\delta X_{, i}^{A}=0$. 
which, together with (3.2), when substituted into the first of (3.1), after using the definitions of stress and couple resultants in the form

$$
\left\{\begin{array}{l}
N^{\alpha \beta} \\
M^{\alpha \beta}
\end{array}\right\}=\int_{-h / 2}^{h / 2}(g / a)^{1 / 2} \sigma^{\alpha \lambda} \mu_{\lambda}^{\beta}\left\{\begin{array}{c}
1 \\
x^{3}
\end{array}\right\} d x^{3},
$$

yields

$$
\delta U=\frac{1}{2} \int_{.}\left\{\left[N^{\lambda \eta}-b_{\alpha}^{\lambda} M^{\alpha \eta}\right] \delta a_{\lambda \eta}-2 M_{. \lambda}^{\beta} \delta b_{\beta}^{\lambda}\right\} d s,
$$

where again (2.8) has been used.

In view of the second of (2.3) and since under the Kirchhoff hypothesis $h=$ const., if we define

$$
\rho=\int_{-h / 2}^{h / 2}(g / a)^{1 / 2} \rho^{*} d x^{3}, \quad \rho_{0}=\int_{-k / 2}^{h / 2}(G / A)^{1 / 2} \rho_{0}^{*} d x^{3},
$$

as the mass density at time $t$ and the initial mass density, both per unit area of the middle surface, it then follows from the law of conservation of mass in the form

$$
\frac{\rho^{*}}{\rho_{0}^{*}}=\left(\frac{G}{g}\right)^{1 / 2}\left|x_{, A}^{i}\right|
$$

that

$$
\frac{\rho}{\rho_{0}}=\left(\frac{A}{a}\right)^{1 / 2}\left|x_{. \Delta}^{\alpha}\right|
$$

Recalling the expression for the total strain energy of the shell space, namely

$$
U=\int_{v} \frac{\rho^{*}}{\rho_{0}^{*}} \Sigma^{*}\left(e_{i j}\right) d v=\int_{V} \Sigma^{*}\left(E_{A B}\right) d V,
$$

then with the aid of (3.2) and (3.7)

$$
U=\int_{S} \int_{-h / 2}^{h / 2}(g / a)^{1 / 2}(G / g)^{1 / 2}\left|x_{, A}^{i}\right| \Sigma^{*} d x^{3} d s
$$

and by (3.8)

$$
U=\int_{s} \frac{\rho}{\rho_{0}} \Sigma d s=\int_{S} \Sigma d S
$$

where

$$
\Sigma=\int_{-h / 2}^{h / 2}(G / A)^{1 / 2} \Sigma^{*}\left(E_{\Delta \Gamma}\right) d x^{3}
$$

is the strain energy density per unit area of the middle surface.

Next, utilizing (3.10), we write

$$
\delta U=\int_{\mathrm{s}} \frac{\rho}{\rho_{0}} \delta \Sigma d s=\int_{\mathrm{s}} \frac{\rho}{\rho_{0}}\left[\frac{\partial \Sigma}{\partial{ }_{0} e_{\alpha \beta}} \delta_{0} e_{\alpha \beta}+\frac{\partial \Sigma}{\partial{ }_{1} e_{\alpha \beta}} \delta_{1} e_{\alpha \beta}+\frac{\partial \Sigma}{\partial{ }_{2} e_{\alpha \beta}} \delta_{2} e_{\alpha \beta}\right] d s .
$$

But from (2.12) we have 


$$
\begin{gathered}
2 \delta_{0} e_{\alpha \beta}=\delta a_{\alpha \beta}, \\
2 \delta_{1} e_{\alpha \beta}=-2 \delta b_{\alpha \beta}=-\delta\left(a_{\alpha \lambda} b_{\beta}^{\lambda}+a_{\beta \lambda} b_{\alpha}^{\lambda}\right) \\
=-\left[b_{\beta}^{\lambda} \delta a_{\alpha \lambda}+b_{\alpha}^{\lambda} \delta a_{\beta \lambda}+a_{\alpha \lambda} \delta b_{\beta}^{\lambda}+a_{\beta \lambda} \delta b_{\alpha}^{\lambda}\right], \\
2 \delta_{2} e_{\alpha \beta}=\delta\left(b_{\alpha \lambda} b_{\beta}^{\lambda}\right)=\delta\left(a_{\lambda \eta} b_{\alpha}^{\eta} b_{\beta}^{\lambda}\right)=b_{\alpha}^{\eta} b_{\beta}^{\lambda} \delta a_{\lambda \eta}+b_{\alpha \lambda} \delta b_{\beta}^{\lambda}+b_{\beta \lambda} \delta b_{\alpha}^{\lambda},
\end{gathered}
$$

the substitution of which into (3.12) results in

$$
\begin{aligned}
\delta U=\frac{1}{2} \int_{0} & \frac{\rho}{\rho_{0}}\left\{\left[\frac{\partial \Sigma}{\partial_{0} e_{\lambda_{\eta}}}-b_{\beta}^{\lambda} \frac{\partial \Sigma}{\partial_{1} e_{\eta \beta}}-b_{\alpha}^{\eta} \frac{\partial \Sigma}{\partial_{1} e_{\alpha \lambda}}\right.\right. \\
& \left.+b_{\alpha}^{\eta} b_{\beta}^{\lambda} \frac{\partial \Sigma}{\partial{ }_{2} e_{\alpha \beta}}\right] \delta a_{\lambda \eta}+\left[-a_{\alpha \lambda} \frac{\partial \Sigma}{\partial{ }_{1} e_{\alpha \beta}}\right. \\
& \left.\left.-a_{\rho \lambda} \frac{\partial \Sigma}{\partial_{1} e_{\beta \rho}}+b_{\alpha \lambda} \frac{\partial \Sigma}{\partial{ }_{2} e_{\alpha \beta}}+b_{\rho \lambda} \frac{\partial \Sigma}{\partial_{2} e_{\beta \rho}}\right] \delta b_{\beta}^{\lambda}\right\} d s .
\end{aligned}
$$
Hence,

In order to obtain the desired results, it will suffice to compare (3.5) and (3.14). ${ }^{9}$

$$
\begin{aligned}
N^{\lambda \eta}-b_{\alpha}^{\lambda} M^{\alpha \eta} & =\frac{\rho}{\rho_{0}}\left[\frac{\partial \Sigma}{\partial{ }_{0} e_{\lambda \eta}}-b_{\beta}^{\lambda} \frac{\partial \Sigma}{\partial{ }_{1} e_{\eta \beta}}-b_{\alpha}^{\eta} \frac{\partial \Sigma}{\partial{ }_{1} e_{\alpha \lambda}}+b_{\alpha}^{\eta} b_{\beta}^{\lambda} \frac{\partial \Sigma}{\partial{ }_{2} e_{\alpha \beta}}\right] \\
M_{. \lambda}^{\beta} & =\frac{\rho}{\rho_{0}}\left[\frac{\partial \Sigma}{\partial_{1} e_{\alpha \beta}} a_{\alpha \lambda}-\frac{\partial \Sigma}{\partial_{2} e_{\alpha \beta}} b_{\alpha \lambda}\right],
\end{aligned}
$$

where it should be noted that the right-hand side of the first of (3.15) is symmetric and satisfies the equation of moment equilibrium about the normal to the deformed middle surface. From the combination of (3.15) and after simplification, there follows the constitutive relations

$$
\begin{aligned}
N^{\beta \lambda} & =\frac{\rho}{\rho_{0}}\left[\frac{\partial \Sigma}{\partial_{0} e_{\beta \lambda}}-b_{\alpha}^{\lambda} \frac{\partial \Sigma}{\partial_{1} e_{\alpha \beta}}\right], \\
M^{\beta \lambda} & =\frac{\rho}{\rho_{0}}\left[\frac{\partial \Sigma}{\partial_{1} e_{\beta \lambda}}-b_{\alpha}^{\lambda} \frac{\partial \Sigma}{\partial_{2} e_{\alpha \beta}}\right]
\end{aligned}
$$

which may alternatively be expressed in terms of $\Sigma\left(E_{\Delta \mathrm{r}}\right)$. For this purpose, observing that by (2.15)

$$
\frac{\partial \Sigma}{\partial_{n} e_{\alpha \beta}}=x_{, \Delta}^{\alpha} x_{, \Gamma}^{\beta} \frac{\partial \Sigma}{\partial{ }_{n} e_{\Delta \mathrm{r}}} ; \quad(n=0,1,2),
$$

we have

$$
\begin{aligned}
& N^{\beta \lambda}=\frac{\rho}{\rho_{0}}\left[\frac{\partial \Sigma}{\partial_{0} E_{\Delta \Gamma}} \delta_{\alpha}^{\lambda}-b_{\alpha}^{\lambda} \frac{\partial \Sigma}{\partial_{1} E_{\Delta \Gamma}}\right] x_{, \Delta}^{\alpha} x_{, \Gamma}^{\beta}, \\
& M^{\beta \lambda}=\frac{\rho}{\rho_{0}}\left[\frac{\partial \Sigma}{\partial_{1} E_{\Delta \mathrm{r}}} \delta_{\alpha}^{\lambda}-b_{\alpha}^{\lambda} \frac{\partial \Sigma}{\partial_{2} E_{\Delta \Gamma}}\right] x_{,{ }_{,}} x_{,{ }_{\mathrm{r}},},
\end{aligned}
$$

If prior to the comparison of (3.5) and (3.14) we first symmetrize $\delta b_{\beta}^{\lambda}$ and write $\delta b_{\alpha \beta}$, then the procedure would be similar to that used by Sanders [8] who also employs different definitions for stress and couple resultants. 
We also note here that in a convected coordinate system $\left(x_{, \Delta}^{\alpha}=\delta_{\Delta}^{\alpha}\right)$, the above constitutive equations may be written as

$$
\begin{aligned}
N^{\beta \lambda} & =\frac{\rho}{\rho_{0}}\left[\frac{\partial \Sigma}{\partial_{0} E_{\beta \lambda}}-b_{\alpha}^{\lambda} \frac{\partial \Sigma}{\partial_{1} E_{\alpha \beta}}\right], \\
M^{\beta \lambda} & =\frac{\rho}{\rho_{0}}\left[\frac{\partial \Sigma}{\partial_{1} E_{\beta \lambda}}-b_{\alpha}^{\lambda} \frac{\partial \Sigma}{\partial_{2} E_{\alpha \beta}}\right] .
\end{aligned}
$$

The constitutive equatious (3.17), derived under the Kirchhoff hypothesis, characterize the mechanical behaviour of elastic shells by a single scalar $\Sigma$ defined through (3.11). ${ }^{10}$ More explicit forms of the constitutive relations (3.17) may be deduced by restricting the functional form of $\Sigma$. We do not pursue this aspect of the subject here, but merely note that for isotropic materials, on the basis of the works of Rivlin and Ericksen [12] and Rivlin [13], $\Sigma$ may be expressed in terms of scalar invariants of four $2 \times 2$ matrices consisting of $B_{\Delta}^{\Gamma}$ and the three submeasures of strain.

4. Alternate derivation of the constitutive equations. Reduction to the linear theory. In this section, we derive the constitutive equations for shells by direct integration with respect to $x^{3}$ of those of the nonlinear theory of hyperelastic materials in 3-space, i.e.,

$$
\sigma^{i i}=\frac{\rho^{*}}{\rho_{0}^{*}} \frac{\partial \Sigma^{*}}{\partial e_{i i}}=\frac{\rho^{*}}{\rho_{0}^{*}} \frac{\partial \Sigma^{*}}{\partial E_{A B}} x_{, A}^{i} x_{, B}^{i},
$$

and then briefly discuss the reduction of the resulting expressions to those of the linear theory.

In view of the structure of (2.11), first observe that

$$
\frac{\partial \Sigma^{*}}{\partial_{n} e_{\alpha \beta}}=\left(x^{3}\right)^{n} \frac{\partial \Sigma^{*}}{\partial e_{\alpha \beta}} ; \quad(n=0,1,2) .
$$

Next, by (3.4), (4.1), (3.7), and (2.8), the stress resultants may be expressed as

$$
N^{\alpha \beta}=\int_{-h / 2}(g / a)^{1 / 2}(G / g)^{1 / 2}\left|x_{, \Delta}^{\alpha}\right| \frac{\partial \Sigma^{*}}{\partial e_{\alpha \rho}}\left(\delta_{\rho}^{\beta}-x^{3} b_{\rho}^{\beta}\right) d x^{3}
$$

which, after using (4.2) and (3.8) and invoking (3.11), yields the first of (3.16). The second of (3.16) may be obtained in a similar manner from the second of (3.4).

In the remainder of this section, we consider the reduction of the constitutive relations derived in Sec. 3 to the corresponding results in the linear theory of elastic shells. First, we note that for the linear theory, (3.11) may be replaced by

$$
\Sigma=\int_{-h / 2}^{h / 2}(g / a)^{1 / 2} \Sigma^{*} d x^{3}
$$

so that in view of (2.11),

$$
\frac{\partial \Sigma}{\partial_{n} e_{\alpha \beta}}=\int_{-h / 2}^{h / 2}(g / a)^{1 / 2} \frac{\partial \Sigma^{*}}{\partial e_{\alpha \beta}}\left(x^{3}\right)^{n} d x^{3} ; \quad(n=0,1,2) .
$$

Further, linearization of (2.5) yields

$$
2 e_{\alpha \beta}=\mu_{\alpha}^{\gamma}\left(\phi_{\gamma \beta}+x^{3} \kappa_{\gamma \beta}\right)+\mu_{\beta}^{\gamma}\left(\phi_{\gamma \alpha}+x^{3} \kappa_{\gamma \alpha}\right)
$$

${ }^{10} \mathrm{At}$ least in principle, for a given $\Sigma^{*}\left(E_{\Delta \Gamma}\right)$ in 3-space, (3.11) determines the corresponding $\Sigma$. 
with the aid of which

$$
\frac{\partial \Sigma^{*}}{\partial \phi_{\alpha \beta}}=\frac{1}{2} \frac{\partial \Sigma^{*}}{\partial e_{\lambda \eta}}\left[\mu_{\lambda}^{\gamma} \delta_{\gamma}^{\alpha} \delta_{\eta}^{\beta}+\mu_{\eta}^{\gamma} \delta_{\gamma}^{\alpha} \delta_{\lambda}^{\beta}\right]=\mu_{\lambda}^{\alpha} \frac{\partial \Sigma^{*}}{\partial e_{\lambda \beta}}
$$

and similarly,

$$
\frac{\partial \Sigma^{*}}{\partial \kappa_{\alpha \beta}}=x^{3} \mu_{\lambda}^{\alpha} \frac{\partial \Sigma^{*}}{\partial e_{\lambda \beta}}
$$

Introducing (4.4) into (3.16), recalling that for the linear theory $\rho / \rho_{0} \simeq 1$, making use of (4.6) and invoking (4.3), there results

$$
N^{\beta \lambda}=\frac{\partial \Sigma}{\partial \phi_{\lambda \beta}}, \quad M^{\beta \lambda}=\frac{\partial \Sigma}{\partial \kappa_{\lambda \beta}}
$$

which are the same as those appropriate for the linear theory under the Kirchhoff hypothesis [10; Sec. 6]. The more explicit form of the linear constitutive equations for shells may be deduced by further assuming a quadratic form for $\sum^{*}\left(e_{\alpha \beta}\right)$ in (4.3).

5. Approximations: Axisymmetric deformation of shells of revolution. As may be seen from the results of Secs. 2 and 3, the exact and general equations characterizing the deformation of an elastic shell, even under the Kirchhoff hypothesis, are still fairly complex and discouraging from the point of view of application. For this reason and in order to render the nonlinear equations of shell theory more manageable, previous investigators (e.g., $[6,7,8,9])$, in addition to the use of generalized Hooke's law, have introduced other plausible assumptions and useful approximations, especially in the kinematic description of the problem. Although these further assumptions are often justified from physical considerations, nevertheless it is desirable to isolate their effect and consequent approximations and, if possible, to indicate precisely the position of the approximate theory relative to the exact. With this in mind and motivated by Reissner's contributions, in the remainder of this paper we confine attention to axisymmetric deformation of shells of revolution and attempt to trace and identify the nature of the approximations in [6].

For ready comparison, we adopt here the notation employed by Reissner [6, 7] and, for the square of linear elements under the Kirchhoff hypothesis and in revolution coordinates, we write

$$
\begin{aligned}
d \mathbf{R}^{*} \cdot d \mathbf{R}^{*} & =\left[\alpha_{0}\left(1-X^{3} \frac{\phi_{0}^{\prime}}{\alpha_{0}}\right)\right]^{2} d\left(X^{1}\right)^{2}+\left[r_{0}\left(1-X^{3} \frac{\sin \phi_{0}}{r_{0}}\right)\right]^{2} d\left(X^{2}\right)^{2}+d\left(X^{3}\right)^{2}, \\
d \mathbf{r}^{*} \cdot d \mathbf{r}^{*} & =\left[\alpha\left(1-X^{3} \frac{\phi^{\prime}}{\alpha}\right)\right]^{2} d\left(X^{1}\right)^{2}+\left[r\left(1-X^{3} \frac{\sin \phi}{r}\right)\right]^{2} d\left(X^{2}\right)^{2}+d\left(X^{3}\right)^{2},
\end{aligned}
$$

where prime designates differentiation with respect to $X^{1}$ (denoted by $\xi$ in [6]) and the quantities to which a subscript 0 is attached refer to the undeformed middle surface. By (2.14) and from the difference of the two expressions in (5.1), we have

$$
\begin{aligned}
2\left[{ }_{0} E_{\Delta \Gamma}\right. & \left.+X^{3}{ }_{1} E_{\Delta \Gamma}+\left(X^{3}\right)^{2}{ }_{2} E_{\Delta \Gamma}\right] d X^{\Delta} d X^{\Gamma} \\
& =\left[\left(\alpha^{2}-\alpha_{0}^{2}\right)-2 X^{3}\left(\alpha \phi^{\prime}-\alpha_{0} \phi_{0}^{\prime}\right)+\left(X^{3}\right)^{2}\left({\phi^{\prime}}^{2}-\phi_{0}^{\prime 2}\right)\right] d\left(X^{1}\right)^{2} \\
& +\left[\left(r^{2}-r_{0}^{2}\right)-2 X^{3}\left(r \sin \phi-r_{0} \sin \phi_{0}\right)\right. \\
& \left.+\left(X^{3}\right)^{2}\left(\sin ^{2} \phi-\sin ^{2} \phi_{0}\right)\right] d\left(X^{2}\right)^{2}
\end{aligned}
$$


which supplies the following submeasures of strain:

$$
\begin{gathered}
{ }_{0} E_{\Delta \Gamma}=\frac{1}{2}\left[\begin{array}{cc}
\left(\alpha^{2}-\alpha_{0}^{2}\right) & 0 \\
0 & \left(r^{2}-r_{0}^{2}\right)
\end{array}\right], \quad{ }_{1} E_{\Delta \Gamma}=-\left[\begin{array}{cc}
\left(\alpha \phi^{\prime}-\alpha_{0} \phi_{0}^{\prime}\right) & 0 \\
0 & \left(r \sin \phi-r_{0} \sin \phi_{0}\right)
\end{array}\right], \\
{ }_{2} E_{\Delta \Gamma}=\frac{1}{2}\left[\begin{array}{cc}
\left({\phi^{\prime}}^{2}-{\phi_{0}^{\prime 2}}^{2}\right) & 0 \\
0 & \left(\sin ^{2} \phi-\sin ^{2} \phi_{0}\right)
\end{array}\right] .
\end{gathered}
$$

It is expedient for future reference to carry out the subsequent discussion in terms of the physical components of strain. To this end, recalling that the quantities which appear in the constitutive equations (3.17) are surface tensors, we calculate the physical components of (5.3) with respect to the metric of the undeformed middle surface $A_{\Delta \mathrm{r}}$ (rather than $G_{\Delta \Gamma}$ ) and obtain

$$
\begin{array}{r}
{ }_{0} E_{(11)}=\frac{1}{2} \frac{\alpha^{2}-\alpha_{0}^{2}}{\alpha_{0}^{2}}, \quad{ }_{0} E_{(22)}=\frac{1}{2} \frac{r^{2}-r_{0}^{2}}{r_{0}^{2}}, \quad{ }_{0} E_{(\cdot 0)}={ }_{0} E_{(21)}=0, \\
{ }_{1} E_{(11)}=-\frac{\alpha \phi^{\prime}-\alpha_{0} \phi_{0}^{\prime}}{\alpha_{0}^{2}}, \quad{ }_{1} E_{(22)}=-\frac{r \sin \phi-r_{0} \sin \phi_{0}}{r_{0}^{2}}, \\
{ }_{1} E_{(12)}={ }_{1} E_{(21)}=0, \\
{ }_{2} E_{(11)}=\frac{1}{2} \frac{\phi^{\prime 2}-\phi_{0}^{\prime 2}}{\alpha_{0}^{2}}, \quad{ }_{2} E_{(22)}=\frac{1}{2} \frac{\sin ^{2} \phi-\sin ^{2} \phi_{0}}{r_{0}^{2}}, \quad{ }_{2} E_{(12)}={ }_{2} E_{(21)}=0 .
\end{array}
$$

Consider now the first of (5.4a) and the first of (5.4b), which may be written as

$$
{ }_{0} E_{(11)}=\frac{\alpha-\alpha_{0}}{\alpha_{0}}\left[1+\frac{\alpha-\alpha_{0}}{2 \alpha_{0}}\right], \quad{ }_{1} E_{(11)}=-\frac{1}{\alpha_{0}}\left[\phi^{\prime}\left(1+\frac{\alpha-\alpha_{0}}{\alpha_{0}}\right)-\phi_{0}^{\prime}\right] .
$$

Clearly, if $\left[\left(\alpha-\alpha_{0}\right) / \alpha_{0}\right] \ll 1$, then by (5.5)

$$
{ }_{0} E_{(11)}=\frac{\alpha-\alpha_{0}}{\alpha_{0}}, \quad{ }_{1} E_{(11)}=-\frac{\phi^{\prime}-\phi_{0}^{\prime}}{\alpha_{0}},
$$

both subject to the same restriction which may be stated as ${ }_{0} E_{(11)} \ll 1$. Similarly, if $\left[\left(r-r_{0}\right) / r_{0}\right] \ll 1$, by the remaining nonvanishing strains in $(5.4 \mathrm{a}, \mathrm{b})$

$$
{ }_{0} E_{(22)}=\frac{r-r_{0}}{r_{0}}, \quad{ }_{1} E_{(22)}=-\frac{\sin \phi-\sin \phi_{0}}{r_{0}},
$$

and again both subject to the same identical restriction ${ }_{0} E_{(22)} \ll 1$.

The submeasures of strain in the forms (5.6) and (5.7) are identical to those derived in a different manner by Reissner [6] who, with the limitation to axisymmetric deformation of shells of revolution, ${ }^{11}$ employs Biot's (three-dimensional) strain measure. As pointed out by Truesdell and Toupin [14; Secs. 33 and 33A], Biot's strain measure is not convenient for coordinates other than those along the principal axes of strain, although it is identical to $E_{A B}$ when both are infinitesimal.

The restrictions associated with the derivation of (5.6) and (5.7) lend further support to Reissner's analysis of axisymmetric deformation of shells of revolution with small

\footnotetext{
${ }^{11} \mathrm{As}$ quantities corresponding to ${ }_{2} E_{\Delta \mathrm{\Gamma}}$ do not arise in [6], it may be of interest to note that in the context of the present paper, Reissner's measures may be regarded as equivalent to calculating the physical components of $E_{\Delta \Gamma}$ with respect to $G_{\Delta \Gamma}$ and then approximating $\left(\delta_{\Delta}^{\Gamma}-X^{3} B_{\Delta}^{\Gamma}\right)$ by $\delta_{\Delta}^{\Gamma}$ in the denominator of the resulting expressions.
} 
middle surface strains $\left({ }_{0} E_{(\Delta \Gamma)} \ll 1\right)$; the additional submeasure of strain ${ }_{2} E_{\Delta \mathrm{r}}$ will lead to terms of higher order in $h / R$ in the constitutive equations and should not be necessary in most practical problems. Furthermore, the assumption ${ }_{0} E_{(\Delta \Gamma)} \ll 1$ is in harmony with the initial Kirchhoff hypothesis, since for small middle surface strains any change in thickness is likely to be negligible. In this connection, it is of interest to note that for finite ${ }_{0} E_{\Delta \mathrm{r}}$, the formulation of the membrane theory of elastic shells, as given by Green and Adkins [15], does account for thickness change.

\section{REFERENCES}

1. J. L. Synge and W. Z. Chien, The intrinsic theory of elastic shells and plates, Th. v. Karman Anniv. Vol., 103, 1941

2. W. Z. Chien, The intrinsic theory of thin shells and plates, Quart. Appl. Math. 1 (1944) 297; 2 (1944) 43 and 120

3. P. M. Naghdi, A survey of recent progress in the theory of elastic shells, Appl. Mech. Reviews 9 (1956) 365

4. J. L. Ericksen and C. Truesdell, Exact theory of stress and strain in rods and shells, Arch. Ratl. Mech. Anal. 1 (1958) 295

5. V. V. Novozhilov, Foundations of the nonlinear theory of elasticity (translated from the first 1948 Russian edition), Graylock Press, 1953.

6. E. Reissner, On the theory of thin elastic shells, H. Reissner Anniversary Volume, J. W. Edwards, Ann Arbor, Mich., 231, 1949

7. E. Reissner, On axisymmetrical deformations of shells of revolution, Proc. Symposia Appl. Math. 3 (1950) 27

8. J. L. Sanders, Jr., Nonlinear theories of thin shells, Tech. Rept. No. 10, Contract Nonr 1866(02), Harvard University, 1961

9. R. W. Leonard, Nonlinear first approximation thin shell and membrane theory, NASA, 1961, to appear.

10. P. M. Naghdi, Foundations of elastic shell theory, Tech. Rept. No. 15, Contract Nonr 222(69), University of California, Berkeley, to appear

11. A. E. Green and W. Zerna, Theoretical elasticity, Oxford, Clarendon Press, 1954

12. R. S. Rivlin and J. L. Ericksen, Stress-deformation relations for isotropic materials, J. Ratl. Mech. Anal. 4 (1955) 323

13. R. S. Rivlin, Further remarks on the stress-deformation relations for isotropic materials, J. Ratl. Mech. Anal. 4 (1955) 681

14. C. Truesdell and R. Toupin, The classical field theories, Handbuch der Physik III/1, 226, 1960

15. A. E. Green and J. E. Adkins, Large elastic deformations, Oxford, Clarendon Press, 1960 